\title{
Study on a Certain Type of Equipment Order Device Test System
}

\author{
Fan Zhenqin, Yang Lei, Yang Xiaolong, Xiong Runze \\ No. 63981 Unit of PLA, Wuhan, China \\ email: phoe_nirvana@163.com
}

\begin{abstract}
According to a certain type of equipment, an order device test system is designed. The test system can totally accomplish whole machine performance test. For its handy operation, the time for equipment test and troubleshooting is greatly shortened, while the field battle mobile support capacity of this typed missile equipment is improved effectively.
\end{abstract}

Keywords: order device; test system; equipment test, mobile support.

\section{Introduction}

A certain type of equipment is largely adopt in army and usually used in military maneuvers. For this, its maintenance support is especially important. Among all the tasks of fault detection, test of order device is the key part, for its limited test measurements and complex operation. In current, the test and maintenance of order device can only be done by MP test and MS test in second grade maintenance. But the process of second grade maintenance is very complex and cost too much time. It has become harder to adapt the fast, accuracy and comprehensive need for the information-based equipment support. And the equipment support capacity and usual training is limited hardly. To solve this problem, a certain type of order system device test system is designed in this paper. For its handy operation, this test system can adapt the need of any level maintenance support, especially emergency support. This test system provide powerful test and support methods for the equipment maintenance, combat readiness, emergency support and medium repair of this typed equipment. Time for equipment test and troubleshooting is greatly shortened, while the field battle mobile support capacity of this typed equipment is improved effectively.

\section{Basic principle of order device}

Working process of order device. Working process of order device contains four stages: March, Wait, Gyroscope Start and Launch. The former three stages are reversible, while the Launch stage is irreversible. The working process is shown in Fig. 1. 


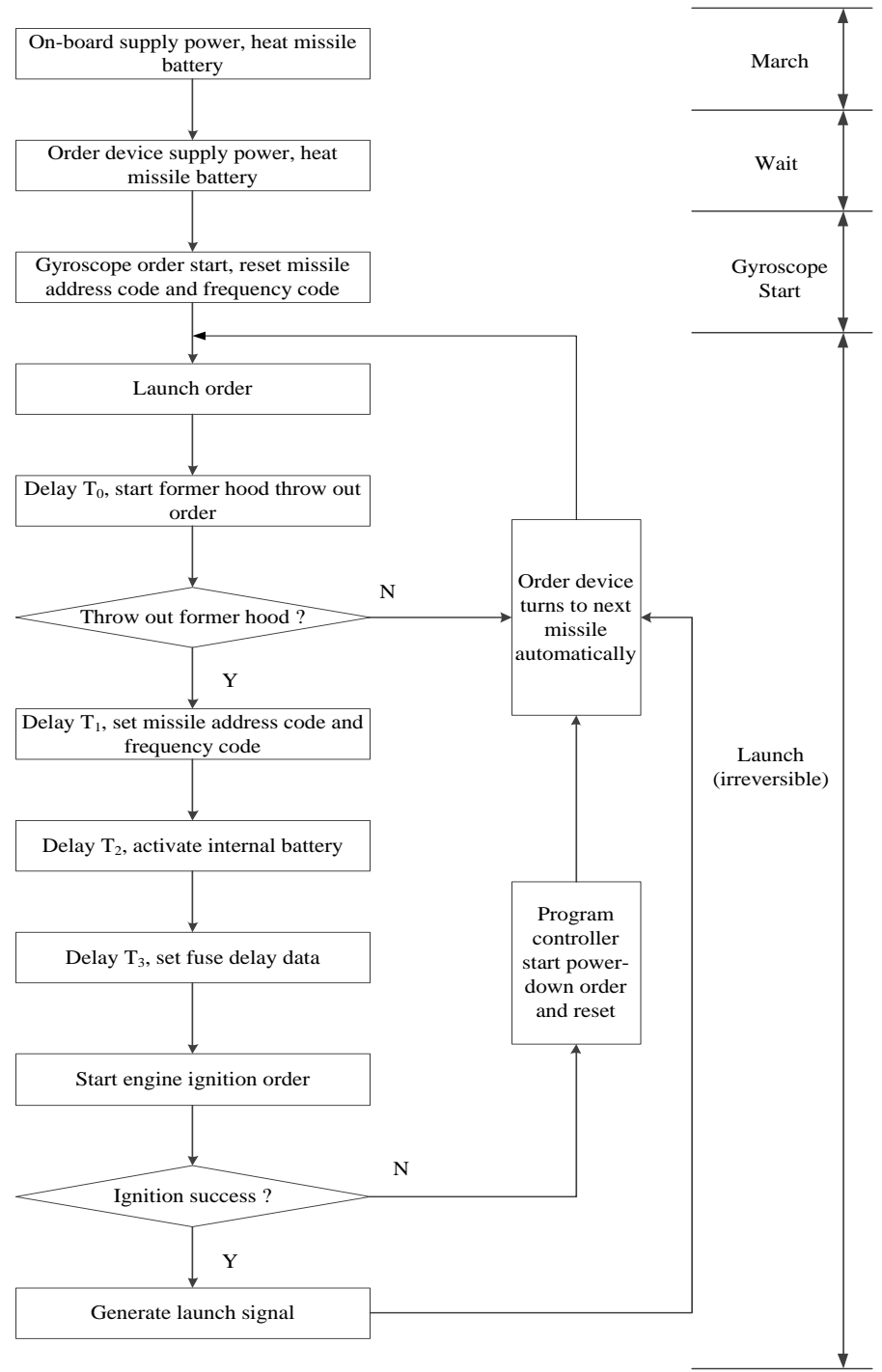

Figure 1. Working process of order device

First, the battery is heated by on-board power supply, then switch to order device power supply. Start gyroscope order and clear address codes and frequency codes to accomplish the initialize settings of launch. On Launch, former hood throw out order is started T0 after the launch button is pushed. Then, the condition of former hood is judged. If the former hood is failed to throw out, the order device switches to next missile automatically. If there is no fault, address codes, frequency codes and fuse delay data will be set after three 
different degree delay time. At last, engine ignition order is started and the condition is judged. If the engine is ignited successfully, the signal will be generated and the missile order device will switch to next one to accomplish single launch. Otherwise, the program controller will start power-down order and reset at first, then switches to next missile.

Order selection. To guarantee that two missiles are ready to launch, the order selection is jointly accomplished by selection combination and launch combination of order device. The principle of order selection is: when the first missile isn't in place, the order device switches to the second missile and starts the third missile's gyroscope at the same time. Likewise, when the second missile isn't in place, the order device switches to the third missile and starts the fourth missile's gyroscope at the same time.

Fault setting. Faults can be set to missile state by the order device. There are two faults: fault of former hood throwing out and fault of engine ignition. When the first fault is set, cannot throw out its former hood in normal. When the last fault is set, missile can throw out its former hood successfully, but failed to ignite the engine.

\section{Design of test system}

System structure. The order device test system contains variable-frequency power supply, test console, strip-chart recorder, multimeter, acquisition card, computer, printer and test cables. The system structure is shown in Fig. 2.

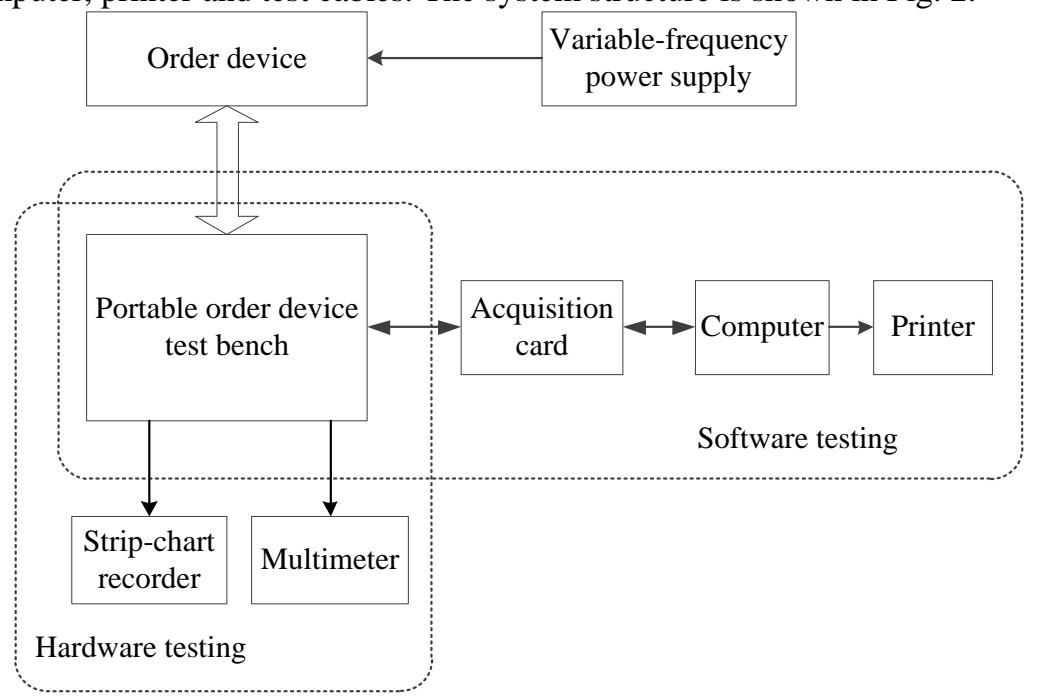

Figure 2. Working process of equipment order device.

Design of system software. The software of test system is developed by LabVIEW. It is mainly used to configure and control hardware resources of test platform. According to the need of fault diagnose, it can accomplish signal collection, data processing, report generation etc. The test program contains 
automatic test program, data management program and fault analysis program. Its running process is shown in Fig. 3.

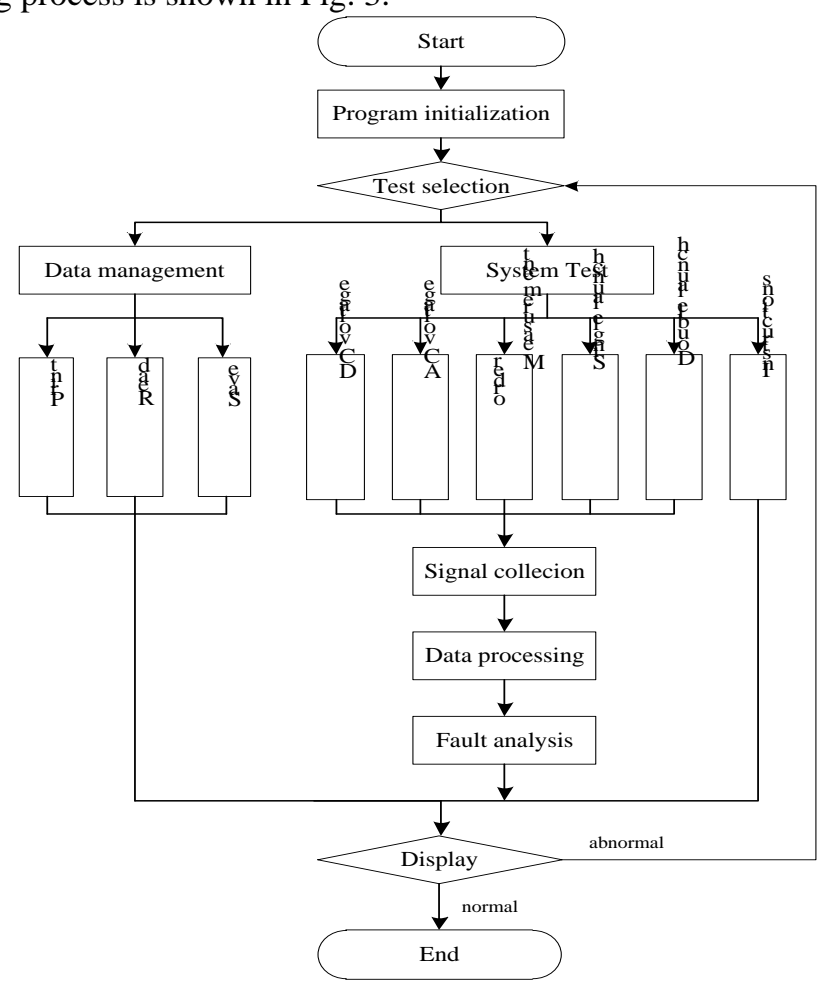

Figure 3. Program running diagram

\section{Data Processing}

The need of missile order device's signal output and its measurement accuracy is very high. Despite of the necessary hardware measure, software measure like digital filter is also adopt in system to improve test accuracy.

Program-judgment filtering. When the sampling signals are affected by random interruption, error detection or unstable of sensor, program-judgment filter can be adapted to solve the signal distortion.

According to the production experience, program-judgment filter determines the probable maximum difference $\Delta y_{s}$ between two sampling output signals. If the real difference is bigger than the probable one, the input signal contains interruption and should be rejected. Otherwise, the input signal should be accepted as sampling value. The concrete process is to determine the sampling result $y_{N}$ by compare $\Delta y_{s}$ with absolute value between the n-th sampling value and its former sampling value. 
If $|y(N)-y(N-1)| \geq \Delta y_{s} \quad, \quad$ then $\quad y_{N}=y(N) \quad$;

(1)

If $|y(N)-y(N-1)| \leq \Delta y_{s} \quad, \quad$ then $\quad y_{N}=y(N-1) \quad$; (2)

In the type: $y(N)$ and $y(N-1)$ are the nth sampling value and its former sampling value, and $y(N)$ is the nth determined sampling result.

The key of this filtering mean is how to choose $\Delta y_{s}$. If $\Delta y_{s}$ is too large, the interruption inhibition ability will be weakened. On the contrary, if $\Delta y_{s}$ is too small, the sampling efficiency will decrease, and the useful signal will be filtered out.

Low-pass filter. Low-pass filter is one kind of kinematic filter, and its real circuit model is RC low-pass filter. The circuit model is shown in Fig. 4.

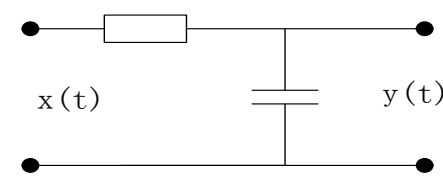

Figure 4. RC low-pass filter model

Let $x(t)$ and $y(t)$ respectively be the input voltage and output voltage of filter, and their relationship is:

$$
R C \bullet \frac{d y(t)}{d t}+y(t)=x(t)
$$

The input voltage and output voltage are sampled for data processing, and their sampling value is:

$$
\begin{aligned}
& x_{n}=x(n \cdot \Delta t) \\
& y_{n}=y(n \cdot \Delta t)
\end{aligned}
$$

(4)

In the type: $\Delta \mathrm{t}$ is sampling interval time; $\mathrm{n}$ is sampling times; $\mathrm{xn}$ and $\mathrm{yn}$ are respectively the input voltage and output voltage sampling value at the first $n$ time.

When the sampling interval time is small enough, the approximate discrete expression is obtained as:

$$
R C \frac{y(n \cdot \Delta t)-y(n-1) \Delta t}{\Delta t}+y(n \cdot \Delta t)=x(n \cdot \Delta t)
$$

That is 


$$
y_{n}=a x_{n}+b y_{n-1} \quad \square \text { or } \square \quad y_{n}=a x_{n}+b y_{n-1}
$$

(6)

$$
\square \text { In the type: } a=\frac{1}{1+\frac{R C}{\Delta t}}, b=\frac{\frac{R C}{\Delta t}}{1+\frac{R C}{\Delta t}} \text {, and } a+b=1 .
$$

This characteristic of this filter is that when the input voltage is DC voltage, there is $x_{n}=y_{n}$ and the gain equals to 1 , for $y_{n}=y_{n}-1$; and when $\Delta \mathrm{t}$ is small enough, there is $a \approx \frac{\Delta t}{R C}$, and the cutoff frequency is:

$$
a=\frac{1}{2^{K}}
$$

The fc changes with the value of a. The lager the value of a is, the higher the fc becomes.

When the constant RC and sampling interval time $\Delta \mathrm{t}$ are set, according to the need of filter, the coefficient $\mathrm{a}$ and $\mathrm{b}$ are determined. The usual value is $a=\frac{1}{2^{K}}$ determined.

\section{Conclusion}

Based on the field battle maintenance support need of certain type equipment, a missile order device test system is designed in this paper. The test system can effectively accomplish whole machine performance test, and greatly shorten the time for equipment test and troubleshooting. It has a very important application value to the field battle mobile and remote support capability of this typed equipment.

\section{Reference}

[1] Wu Xiuqing, Zhou Heqin: Microcomputer theory and interface technology (China Science and Technology Press, China 2001).

[2] Xu Yang: Inertial platform automatic testing system (Beihang University Press, China 2004).

[3] Zhou xingpeng, Qiu Guofu, Wang Shourong: Modern measurement technology (Higher Education Press, China 2004). 
[4] Liu Guoling, Ying Guanxi: Electronic measurement (China Machine Press, China 2003).

[5] Lei Lin: Microcomputer automatic detection and system design (Electronic Industry Press, China 2003).

[6] Chen Guangyu: Modern electronic testing technology (National Defence Industry Press, Chian 2000).

[7] Peng Xuming: Missile order device basic theory and maintenance (China Aerospace Science \& Industry Corp Second Research Institute, China 2004).

[8] Chen Kailiang, Zhu Shusheng: Constant current source and circuit application (Zhejiang Sinence Press, China 1999).

[9] Wu Lixin: Practical electronic technical manual (China Machine Press, China 2002). 